\title{
Genome Wide Identification of FGFR2 Alternative Splicing in Hepatitis C: Potential Roles in Malignant Transformation
}

\section{Sayed S Daoud*}

Department of Pharmaceutical Sciences, Washington State University Health Sciences, Spokane, Washington, USA

\section{Editorial}

Hepatocellular carcinoma (HCC) is one of the few tumors in which the incidence is on the rise worldwide, especially in the US [1]. The increasing incidence of this disease is associated with the rise in hepatitis $\mathrm{C}$ virus (HCV) infection [2]. It is estimated that 3.2 million people in the US are infected with HCV; a blood-borne disease linked to 12,000 US deaths a year [3]. More concerning are projections that rate will quadruple in the next 10 years, to over 40,000 cases per year. Therefore, there is a great need to understand the molecular mechanisms for HCV-induced malignant transformation. Many studies have shown that malignant transformation of HCV infected hepatocytes occurs through a pathway of increased liver cell turnover induced by chronic liver injury and regeneration in a context of inflammation and oxidative DNA damage. In this context, the host immune response against the various $\mathrm{HCV}$-encoded proteins (HCV core, E2, NS3, and NS5A) becomes relevant for HCC development and tumor progression. HCV proteins interact with many host cells factors and affect a wide range of cellular and humoral activities, including cell signaling, transcriptional modulation, transformation, apoptosis, membrane rearrangements, vesicular trafficking and cytokine \& chemokine production. Inflammatory cells and immunomodulatory mediators present in the microenvironment polarize host immune response toward specific phenotypes impacting initiation and progression of the disease [4].

Additional studies also showed that HCV-induced oxidative stress regulates the expression of host-specific genes through alternative splicing (AS), which in turns contributes to the processes of malignant transformation $[5,6]$. Alternative splicing is the key way in which a single gene can produce more than one protein product. It has been recognized that up to $95 \%$ of human genes can give rise to alternatively spliced mRNAs in different human tissues [aka: transcriptome and proteome diversity]. For example, AS resulted from HCV-induced oxidative stress produces multiple protein isoforms, which display various cellular \& biological activities impacting on HCV-induced HCC $[7,8]$. There are a number of distinct mechanisms by which different mRNA may generate isoforms: exon skipping/inclusion, alternative 3' splice sites, alternative 5' splice sties, mutually exclusive exons, and intron retention (Figure 1).

To examine the extent of alternative splicing on transcript regulation of $\mathrm{HCV}$-induced malignant transformation, two recent studies were performed by RNAseq and Human Transcriptome Array (HTA 2.0). In the first study, Lin et al. [9] sequenced 250 million pairend reads from 55 pairs of adjacent normal and tumor liver samples (with various viral status such as $\mathrm{HBV}+\mathrm{HCV}+$ and HBV-, HCV-). Using bioinformatics tools, the authors identified a novel gene with coding regions, termed $D U N Q U 1$, which has a tissue-specific expression pattern in tumor liver samples of $\mathrm{HCV}+$ compared to HCV- and HBVHCC. Further analysis showed that this gene expresses two isoforms (SP1 and SP2), and both have a phosphodiestrases domain and alkaline phosphatase-like domains. Overexpression of DUNQU1 in liver cancer cells enhances the ability to form colonies in soft agar, although it was not clear which isoform has this tumorigenic action. In addition, the authors identified three novel differentially expressed protein-coding genes (ALG1L, SERPINA11 and TMEM82) that lack documented expression profiles in liver cancer. In the same study, the authors observed that AS events are also involved in viral-induced HCC. Three of the AS events (FGFR2, EXOC7 and ADAM15) were found to be cancer related genes. Although AS event of FGFR2 has been shown to be a necessary event during tumorigenesis [10], in this particular case it was not clear which isoform nor the viral status of liver tumor samples. Clearly, this study highlights the clinical importance of genome-wide profiling in the identification of alternative spliced transcripts that could be involved in malignant transformation of viral infection.

In the second study (unpublished) our group has used Affymetrix HTA 2.0 to study the roles of alternative splicing events in HCVmalignant transformation in 16 liver and tumor samples. Using TAC 2.0 software coupled with bioinformatic analyses, we were able to identify about 636 genes that are differentially expressed between normal vs. HCV+ cirrhotic liver samples, and 61 genes were differentially expressed between adjacent normal and tumor liver samples. Some of these genes are novel, and have not been documented to be involved in HCV-induced malignant transformation. For splicing analysis, we have identified 1890 genes with alternative splicing events in the coding regions and 80 in the non-coding regions. As shown in Figure 2, we have identified 9 splice variants in the coding region of FGFR2

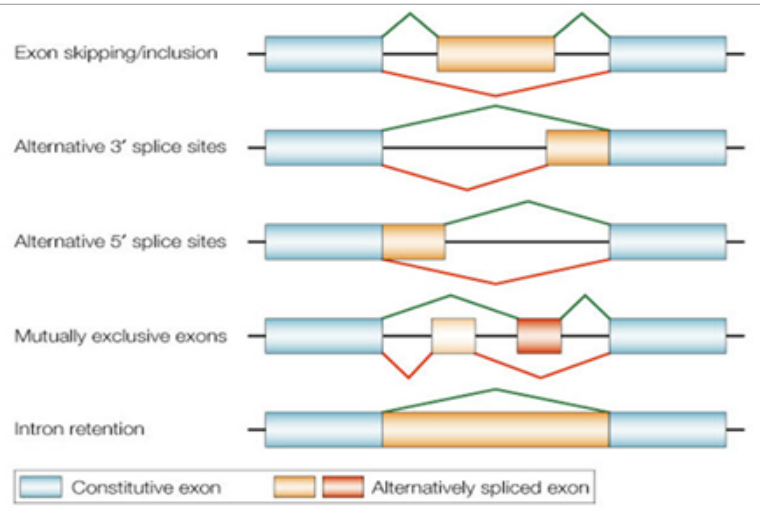

Figure 1: Possible mechanisms of alternative RNA splicing events

*Corresponding author: Sayed S. Daoud, Department of Pharmaceutical Sciences, Washington State University Health Sciences, Spokane, WA, USA, Tel: (509) 3686572; E-mail: daoud@wsu.edu

Received December 16, 2014; Accepted December 17, 2014; Published December 20, 2014

Citation: Daoud SS (2014) Genome Wide Identification of FGFR2 Alternative Splicing in Hepatitis C: Potential Roles in Malignant Transformation. J Pharmacogenomics Pharmacoproteomics 5: e140. doi:10.4172/2153-0645.1000e140

Copyright: (c) 2014 Daoud SS. This is an open-access article distributed under the terms of the Creative Commons Attribution License, which permits unrestricted use, distribution, and reproduction in any medium, provided the original author and source are credited. 
Citation: Daoud SS (2014) Genome Wide Identification of FGFR2 Alternative Splicing in Hepatitis C: Potential Roles in Malignant Transformation. J Pharmacogenomics Pharmacoproteomics 5: e140. doi:10.4172/2153-0645.1000e140

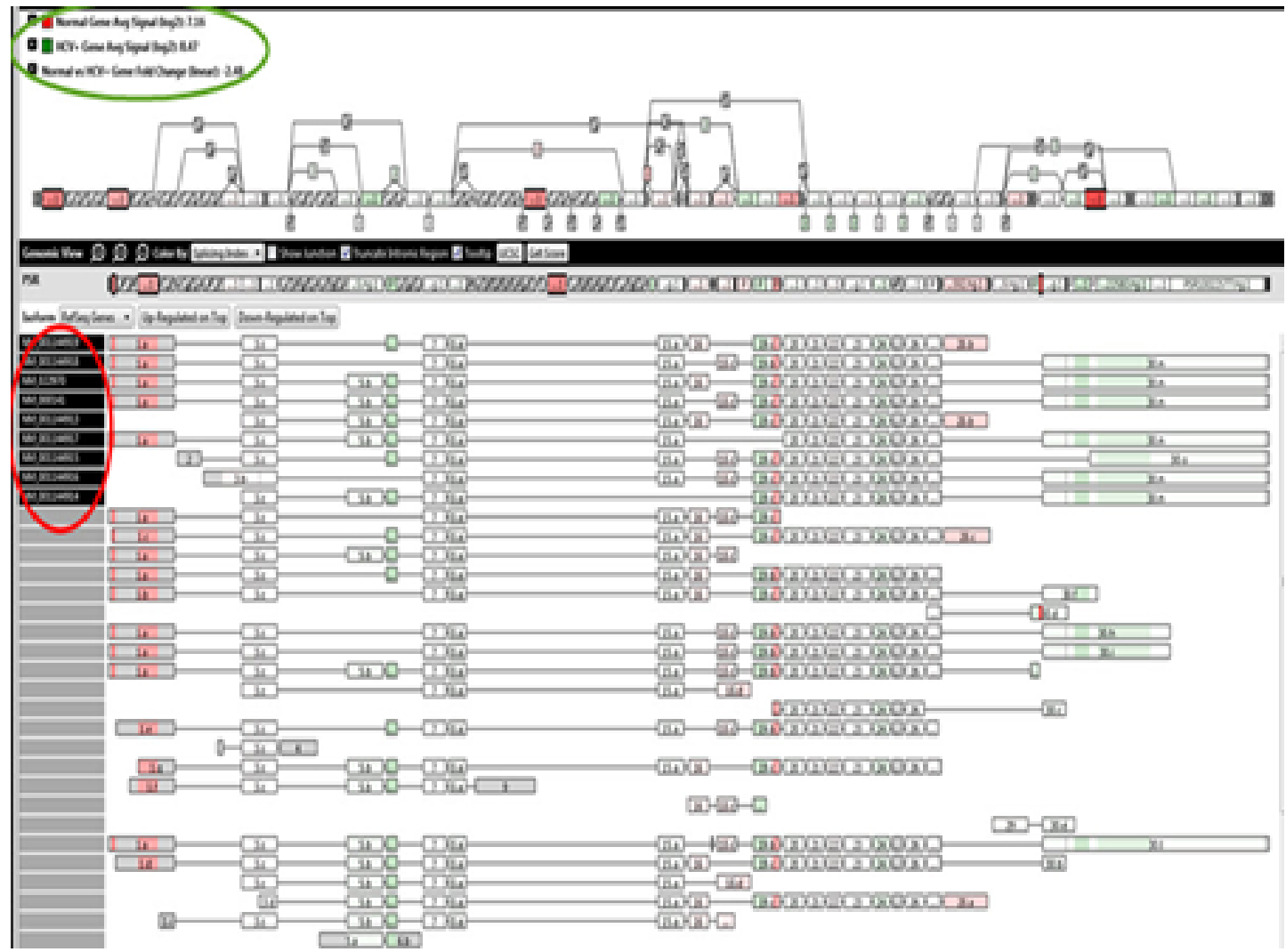

Figure 2: Genomic structure view of FGFR2 splicing events. This view is filtered to show only the accession numbers of isoforms in RefSeq, red label

associated with HCV+ compared to normal liver samples. Although different computational approaches were used, in this study, to identify the splicing events of FGFR2, this showed quite good concordance between the two studies, albeit using two different platforms, at least with respect to one gene. Indeed, follow-up studies are needed to validate the findings in the two projects to understand the molecular basis for $\mathrm{HCV}$-induced malignant transformation.

\section{References}

1. El-Serag HB (2011) Hepatocellular carcinoma: current concepts. N Engl J Med 365:1118-27

2. Edlin BR (2011) Perspective: test and treat this silent killer. Nature 474: S18-S19

3. Jacobson IM, Davis GL, El-Serag H, Negro F, Trépo C (2010) Prevalence and challenges of liver diseases in patients with chronic hepatitis $C$ virus infection. Clin Gastroenterol Hepatol 8:924-33.

4. Castello G, Scala S, Palmieri G, Curley SA, Izzo F (2010) HCV-related hepatocellular carcinoma: From chronic inflammation to cancer. Clin Immunol 134: $237-250$.

5. Hernandez-Lopez HR, Graham SV (2012) Alternative splicing in human tumor viruses: a therapeutic target? Biochem J 445: 145-156.

6. Shawki SM, Meshaal SS, EI Dash AA, Zayed NA, Hanna MO (2014) Increased DNA damage in hepatitis $C$ virus-related hepatocellular carcinoma. DNA Cell Biol 33: 884-890.
7. Hanoun N, Bureau C, Diab T, Gayet O, Dusetti N, et al. (2010) The SV2 variant of KLF6 is downregulated in hepatocellular carcinoma and displays antiproliferative and pro-apoptotic functions. J Hepatol 53: 880-888.

8. Shi Y, Lv G, Chu Z, Piao L, Liu X, et al. (2014) Identification of natural splice variants of SAMHD1 in virus-infected HCC. Oncol Rep 31:687-692.

9. Lin KT, Shann YJ, Chau GY, Hsu CN, Huang CY (2014) Identification of latent biomarkers in hepatocellular carcinoma by ultra-deep whole transcriptome sequencing. Oncogene 33: 4786-4794.

10. Zhao Q, Caballero OL, Davis ID, Jonasch E, Tamboli P, et al. (2013) Tumorspecific isoform switch of the fibroblast growth factor receptor 2 underlies the mesenchymal and malignant phenotypes of clear cell renal cell carcinomas. Clin Cancer Res 19: 2460-2472. 